\title{
Recent advances in the identification of the host factors involved in dengue virus replication
}

\author{
Yi Wang ${ }^{1,2}$, Ping Zhang ${ }^{1,2 \bowtie}$
}

1. Department of Immunology, Institute of Human Virology, Zhongshan School of Medicine, Sun Yat-sen University, Guangzhou 510080, China

2. Key Laboratory of Tropical Diseases Control (Sun Yat-sen University), Ministry of Education, Guangzhou 510080, China

Dengue virus (DENV) belongs to the genus Flavivirus of the family Flaviviridae and it is primarily transmitted via Aedes aegypti and Aedes albopictus mosquitoes. The life cycle of DENV includes attachment, endocytosis, protein translation, RNA synthesis, assembly, egress, and maturation. Recent researches have indicated that a variety of host factors, including cellular proteins and microRNAs, positively or negatively regulate the DENV replication process. This review summarizes the latest findings (from 2014 to 2016) in the identification of the host factors involved in the DENV life cycle and Dengue infection.

\section{KEYWORDS dengue virus (DENV); host factors; replication; proteins; miRNAs}

\section{INTRODUCTION}

The dengue virus (DENV) is a positive-sense singlestranded RNA virus that belongs to family Flaviviridae. Currently, four DENV serotypes have been identified (DENV1-4) (Casseb et al., 2016). All of these serotypes are able to cause a flu-like disease (dengue fever) as well as more severe diseases, such as dengue hemorrhagic fever and dengue shock syndrome (Gubler, 1998). The DENV genome is approximately $11 \mathrm{~kb}$ in length with a $5^{\prime}$ type $\mathrm{I} \mathrm{m}^{7} \mathrm{G}$ cap structure, and it encodes a polyprotein. The polyprotein is post-translationally cleaved by host and viral proteases into three structural proteins (the capsid, C; premembrane/membrane, prM/M; envelope, E) and seven non-structural proteins (NS1, NS2A, NS2B, NS3, NS4A, NS4B, and NS5) (Green et al., 2014).

The DENV virions enter their target cells via receptormediated endocytosis in a clathrin-dependent manner

Received: 3 November 2016, Accepted: 4 January 2017,

Published online: 24 January 2017

$\square$ Correspondence:

Phone: +86-20-87331938, Fax: +86-20-87331938,

Email: zhangp36@mail.sysu.edu.cn

ORCID: 0000-0002-5400-8767
(Acosta et al., 2008; Savidis et al., 2016). In an acidified endosomal vesicle, the virion undergoes conformational changes that enable fusion with the endosomal membrane, releasing the genome RNA into the cytosol (Zaitseva et al., 2010). After the initial translation and cleavage of the polyprotein, DENV triggers the formation of a replication complex in the perinuclear endoplasmic reticulum, where RNA replication and protein translation occur (Welsch et al., 2009). The newly synthesized positivesense RNA is then packaged by the $\mathrm{C}$ protein and assembled into an enveloped virion that is covered with $\mathrm{prM} / \mathrm{E}$ heterodimers. When the vesicles containing the immature virions move through the Golgi apparatus, prM is cleaved by a furin protease. Finally, the immature virions become mature or partially mature virions, which are secreted (Yu et al., 2008).

Research indicates that the ten proteins encoded by the compact DENV genome are insufficient for viral replication. The virus has evolved many strategies to hijack and utilize host factors throughout its life cycle. Host factors have been implicated in the promotion of almost every step of the viral life cycle, including entry, RNA synthesis, protein expression, assembly, egress, and maturation. On the other hand, research has indicated that some host factors protect cells from virus infection and replication. 
Several previous studies summarized the research on the interaction between DENV and host factors (Hidari and Suzuki, 2011; Acosta et al., 2014; Krishnan and Garcia -Blanco, 2014; Salazar et al., 2014). This study reviews the latest findings on host factors that are involved in DENV infection and replication, namely, proteins (Table 1 ) and microRNAs (miRNAs, a class of highly conserved non-coding RNAs of $\sim 22$ nucleotides in length) (Table 2).

\section{HOST PROTEINS INVOLVED IN DENV INFECTION AND REPLICATION}

\section{Proviral proteins}

CD300a. To identify the DENV entry factors, Amara's group carried out a gain-of-function complementary DNA(cDNA) screen for host proteins that allow the minimally susceptible cell line $293 \mathrm{~T}$ to be infectable by DENV (Carnec et al., 2015). In addition to T cell, immunoglobulin, and mucin domain (TIM) and TYRO3, AXL, and MER (TAM) receptor families, CD300a was found to enhance DENV infectivity (Meertens et al., 2012). CD300a is a phospholipid receptor that belongs to the CD300 family, members of which are expressed in many types of immune cells, including all natural killer (NK) cells, subsets of T and B cells, monocytes, dendritic cells, granulocytes, and cord blood -derived mast cells (Borrego, 2013).
In CD300a-expressing 293T cells, the DENV replication levels (including viral RNA, proteins, and particles) were dramatically increased (Carnec et al., 2015). Preincubation of CD300a-expressing cells with a polyclonal anti-CD300a antibody reduced the DENV infection by $80 \%$, indicating that CD300 is a key mediator of viral entry (Carnec et al., 2015). The authors further showed that CD300a directly interacts with phosphatidylethanolamine (PtdEth) on the surface of DENV particles, and promotes viral internalization by clathrin-mediated endocytosis (Carnec et al., 2015). Puzzlingly, although some CD300a-expressing immune cells (such as monocytes and dendritic cells) are susceptible to DENV infection, other CD300a-expressing immune cells (such as NK, T, and $\mathrm{B}$ cells) are not susceptible to DENV infection, indicating that CD300a might be essential, but not sufficient for DENV entry. Nonetheless, the role of CD300a in DENV infection needs to be further investigated using the cells that naturally express CD300a.

Ribonuclease $K$ (RNASEK). Using multiple orthologous RNA interference (RNAi) reagent (MORR) genetic screens, Brass's group found that RNASEK, a transmembrane protein, is required for replication by multiple viruses, including the human rhinovirus, influenza virus, yellow fever virus, and DENVs (Perreira et al., 2015). When RNASEK was depleted using RNAi, the entry of these viruses was blocked and viral replication

Table 1. Host proteins and DENV

\begin{tabular}{|c|c|c|}
\hline Protein & Function & Reference \\
\hline CD300a & $\begin{array}{l}\text { Interacts with the PtdEth on the surface of DENV } \\
\text { particles and promotes viral internalization }\end{array}$ & Carnec et al., 2015 \\
\hline RNASEK & Plays a role in viral endocytosis & Perreira et al., 2015 \\
\hline OST & $\begin{array}{l}\text { Plays a role in viral RNA replication by interacting } \\
\text { with viral proteins }\end{array}$ & Marceau et al., 2016 \\
\hline hnRNP K & Promotes viral multiplication & Brunetti et al., 2015 \\
\hline SPCS & Promotes the processing of structural proteins & Zhang et al., 2016 \\
\hline CCR5 & Facilitates viral infection & Marques et al., 2015 \\
\hline Receptor-activated $\alpha_{2} M\left(\alpha_{2} M^{*}\right)$ & Promotes viral stability and improves viral infectivity & Huerta et al., 2014 \\
\hline GBF1 & Facilitates viral C protein transportation & Iglesias et al., 2015 \\
\hline PEX19 & Signaling platform for innate immunity & You et al., 2015 \\
\hline $\mathrm{HO}-1$ & Inhibits viral NS2B/NS3 protease activity & Tseng et al., 2016 \\
\hline TRIM56 & Inhibits viral RNA replication & Liu et al., 2014 \\
\hline GRP75 & $\begin{array}{l}\text { Promotes mature processing of the antiviral miRNA } \\
\text { hsa-mir-126 }\end{array}$ & Kakumani et al., 2016 \\
\hline elF4Al & Inhibits viral protein synthesis & Chen et al., 2015 \\
\hline DDX3 & Promotes IFN response & Li et al., 2015 \\
\hline
\end{tabular}


Table 2. Host miRNAs and DENV

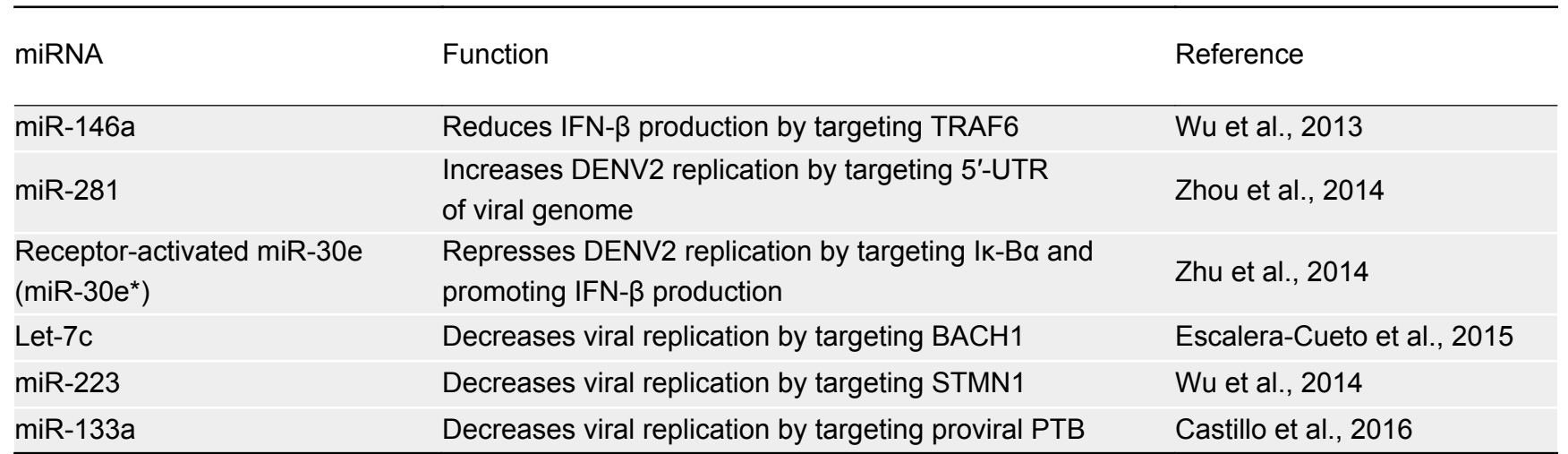

was reduced. By affinity purification coupled with mass spectrometry, the authors identified that RNASEK interacts with subunits of the vacuolar ATPase (VATPase) complex (which is responsible for acidifying intracellular compartments), such as ATP6AP1. Using an RNAi assay, RNASEK was shown to be as important to the maintenance of the endolysosomal acidity as members of the VATPase complex. Depletion of RNASEK led to a decrease in viral endocytosis and the formation of enlarged clathrin-coated pits at the cell surface. Therefore, it is clear that RNASEK plays a crucial role in viral endocytosis by interacting with VATPase (Perreira et al., 2015).

Oligosaccharyltransferase (OST) complex. Using haploid and clustered regularly interspaced short palindromic repeats (CRISPR) /Cas9 genetic screens, Carette's group found that genes encoding several OST complex subunits (STT), which were not previously linked to the replication of any viruses, were the most enriched genes (Marceau et al., 2016). The knockout of the OST catalytic subunits, STT3A or STT3B, in Huh7 cells completely abolished viral RNA replication, while viral entry was unaffected. As the OST complex functions as an enzyme that is essential for N-linked glycosylation, the authors further tested the dependency of the proviral effect of OST on its catalytic activity. They found that STT3A and STT3B mutants that were unable to catalyze glycosylation reactions were able to restore DENV replication in the STT3A- or STT3B-knockout cells, suggesting that DENV hijacks a function of the OST complex other than its glycosylation function. The authors showed that the viral proteins NS2B and NS3 associate specifically with STT3A and STT3B, illustrating that the OST complex plays a role in viral RNA replication by interacting with viral non-structural proteins (Marceau et al., 2016).

Polypyrimidine tract-binding protein (PTB) and heterogeneous nuclear ribonucleoprotein (hnRNP) $K$. The
hnRNPs family consists of 21 proteins (from hnRNP A to hnRNP U) involved in messenger RNA (mRNA) processing, transportation, translation, and stability (Han et al., 2010). So far, several hnRNPs have been shown to be associated with DENV replication. First, hnRNP I (also known as PTB) can bind to the untranslated region (UTR) of viral RNA and NS4A, and it colocalizes with the viral replication complex. Depletion of hnRNP I led to decreased viral RNA synthesis, but it did not affect the translation of viral RNA (Agis-Juarez et al., 2009). Second, hnRNP K has been shown to be associated with DENV replication. Brunetti et al. found that DENV infection led to the cytoplasmic translocation of hnRNP A2 and $\mathrm{K}$, without altering their expression level. The knockdown of hnRNP K resulted in a significant reduction in viral multiplication, while ectopic expression of hnRNAP K did not lead to an increase in viral multiplication, probably because endogenous cytoplasmic hnRNP $\mathrm{K}$ was sufficient to promote viral multiplication (Brunetti et al., 2015).

Signal peptidase complex subunits (SPCSS). Using a CRISPR/Cas9 technique to screen for host factors involved in West Nile virus (WNV) infection, Diamond's group identified a subset of endoplasmic reticulum-associated SPCS proteins that are crucial for WNV replication (Zhang et al., 2016). The knockout of SPCS1 markedly reduced the replication of all the flaviviruses tested, including WNV, DENV2, Japanese encephalitis virus, hepatitis $\mathrm{C}$ virus, yellow fever virus, and Zika virus. In SPCS1-knockout cells, viral RNA translation and replication remained largely intact, but cleavage of the flavivirus structural proteins (prM and E) was greatly impaired. The authors further demonstrated that the viruses in the family Flaviviridae, but not alphavirus, bunyavirus, or rhabdovirus, are dependent on this SPCS-mediated signal peptide processing pathway. Therefore, SPCS1 might play a key role in DENV multiplication by directly or indirectly promoting the processing of Flaviviridae struc- 
tural proteins (Ma et al., 2015; Zhang et al., 2016).

C-C chemokine receptor 5 (CCR5). CCR 5 acts as a receptor for the chemokines CCL3 and CCL5, and it is mainly involved in the recruitment of mononuclear leucocytes to tissues (Oppermann, 2004; Repeke et al., 2010). Based on observations that CCR5 plays a role in the development of inflammatory diseases and the increased levels of CCR5 and CCR5 ligands in DENV patients, Teixeria's group examined the function of CCR5 in DENV infection. DENV infection led to an increased expression of CCR5 ligands such as CCL5 (Marques et al., 2015).

To study the function of CCR5, a CCR5/CCR1 antagonist, Met-RANTES (Met-R), was utilized to block CCR5 activation. Both of pretreatment of macrophages in vitro with Met-R before DENV infection and post-infection treatment with Met-R reduced DENV RNA levels. Although CCR5 and DENV particles become colocalized on the cell membrane, CCR5 was not found to be a receptor for DENV. In a mouse model, the knockout of CCR 5 significantly decreased viral loads in tissues, and the disease severity in the CCR5 KO mice caused by DENV infection was reduced. In addition, preinfection treatment with Met-R reduced the viral load in DENV-infected organs of wild-type mice, but post-infection treatment with Met-R failed to protect the mice (Marques et al., 2015). Although the role of CCR5 in DENV replication has now been well established using in vitro and in vivo infection models, the detailed mechanism of how CCR5 facilitates viral infection requires further elucidation.

Receptor-activated a2-macroglobulin $\left(\alpha_{2} M^{*}\right)$. The glycoprotein homotetramer $\alpha_{2} \mathrm{M}$ acts as a proteinase that can inhibit all protease families (Michael and Salvatore, 1981). Using an affinity chromatography assay, Brasier et al. discovered that $\alpha_{2} \mathrm{M}$ interacts with DENV using domain III of the viral E protein as a ligand (Brasier et al., 2015). The receptor-activated form of $\alpha_{2} \mathrm{M}, \alpha_{2} \mathrm{M}^{*}$ can bind to all four DENV serotypes. The interaction of $\alpha_{2} \mathrm{M}^{*}$ with DENV particles is reversible. An in vitro study showed that the multivalent binding of $\alpha_{2} M^{*}$ to $E$ promoted the structural stabilization of virions from temperature-induced inactivation, and it also improved viral infectivity (Huerta et al., 2014). Interestingly, a quantitative proteomics study identified $\alpha_{2} \mathrm{M}$ as a biomarker for severe DENV infection caused by DENV3 (Brasier et al., 2015). Recently, the application of $\alpha_{2} \mathrm{M}$ for the clinical diagnosis of DENV diseases has been the subject of an international patent filed by the National University of Singapore.
Golgi-associated brefeldin A-resistant guanine nucleotide exchange factor (GBF1). As the newly-synthesized DENV C protein plays a role in viral genome packaging after being transported to cellular lipid droplets, Gamarnik's group investigated role of the lipid dropletassociated proteins in the transportation and accumulation of C protein in lipid droplets (Samsa et al., 2012). They found that the delivery of the $\mathrm{C}$ protein is sensitive to brefeldin A (BFA), an inhibitor of guanosine triphosphate (GTP) exchange factors. GTP-exchange factors such as GBF1 and brefeldin A-inhibited guanine nucleotide-exchange factors 1 and 2 (BIG1, and BIG2) can activate monomeric GTPases by stimulating the release of guanosine diphosphate (GDP) to allow binding of guanosine triphosphate (GTP) (Ramaen et al., 2007). Treatment with BFA markedly decreased the DENV titers. Furthermore, the authors established the important role of GBF 1 in the transportation of the $\mathrm{C}$ protein and viral replication by utilizing a dominant-negative form of GBF1, and small interfering RNA (siRNA) targeting GBF1. The study suggested that GBF1 is a proviral factor of DENV as it facilitates the transportation of the C protein (Iglesias et al., 2015).

\section{Antiviral proteins}

Peroxisome 19 (PEX19). PEX19 is a farnesylated protein that is essential for the biogenesis of peroxisomes, which are an important signaling platform for antiviral innate immunity (Dixit et al., 2010). In an affinity purification screen, PEX19 was found to interact with the C protein of DENV and WNV. In response to DENV infection, PEX19 moved from the perinuclear region to juxtanuclear areas, which were enriched with C protein. DENV infection induced the degradation of the PEX19 protein, leading to a decreased biogenesis of peroxisomes and reduced levels of the peroxisomal matrix enzyme, catalase. Interestingly, expression of the DENV or WNV C protein was able to rescue the interferon (IFN)- $\gamma$ induction that is normally suppressed by DENV and WNV. The authors also showed that PEX19 knockdown by siRNA reduced the production of IFN- $\gamma$ triggered by polyinosinicpolycytidylic acid treatment, suggesting that PEX19 is involved in antiviral innate immunity. However, PEX19 knockdown did not enhance viral replication, as had been predicted, but resulted in reduced replication (You et al., 2015). Therefore, the hypothesis that DENV escapes the peroxisome antiviral effect by recruiting and degrading PEX19 to inhibit peroxisome biogenesis needs to be further validated.

Heme oxygenase-1(HO-1). HO-1, an antioxidant enzyme in the heme catabolic pathway, has a protective effect against inflammatory processes and oxidative tissue damage induced by stimuli such as viral and bacterial 
products (Paine et al., 2010). Lee's group showed that the induction of HO-1 by specific inducers, such as cobalt protoporphyrin (CoPP) and hemin led to a reduction in viral RNA synthesis and the levels of DENV1-4 proteins. These reductions were reversed by treatment with the HO-1 inhibitor tin protoporphyrin IX (SnPP), indicating that HO-1 plays an antiviral role. In DENV-infected Huh-7 cells, treatment with CoPP increased the levels of IFN- $\alpha$ and proteins encoded by interferon-stimulated genes, whereas SnPP treatment attenuated the levels in a dose-dependent manner. Moreover, the authors found that biliverdin, a degraded product of heme, which is catalyzed by HO-1, inhibited the protease activity of the NS2B/NS3 complex, which mediates the cleavage of mediator of IRF3 activation (MITA), an innate adaptor protein. The authors also carried out an in vivo study that involved simultaneously injecting DENV-2 and CoPP into suckling mice, and they found that HO-1 induction delayed DENV2-induced disease and lethality (Tseng et al., 2016). The antiviral role of HO-1 is exerted by its inhibition of DENV protease activity and the restoration of antiviral IFN response.

Tripartite motif-containing 56 (TRIM56). The TRIM protein family consists of more than 70 members. They share three conserved N-terminal domains: a Really Interesting New Gene (RING) domain, one or two BBoxes (B1/B2), and a coiled-coil (CC) domain. Recently, several TRIM members were shown to regulate antiviral innate immune signaling and to possess direct antiviral activities (Rajsbaum et al., 2014). Recently, Li's group reported that TRIM56 plays an antiviral role against yellow fever virus, DENV, and a human coronavirus. Overexpression of TRIM56 led to a decrease in viral protein levels and yield of DENV virions. The antiviral effect of TRIM56 is independent of the innate immune response and regulation of cell growth. The authors mapped the domains of TRIM56 that are responsible for its inhibitory effect, revealing that the E3 ligase activity and the integrity of the C-terminal portion of TRIM56 are indispensable to its anti-DENV function. TRIM56 expression inhibited the luciferase activity of a DENV luciferase reporter replicon, suggesting that TRIM56 plays an antiviral role at the RNA replication stage (Liu et al., 2014). However, the detailed mechanism remains unknown.

$75 \mathrm{kDa}$ glucose-regulated protein (GRP75). As the catalytic components (Dicer, Drosha, AGO1, and AGO2) and an auxiliary component (HSC70) of the host RNAi system have been shown to restrict DENV replication, Bhatnagar's group carried out co-immunoprecipitation assays to identify new cellular components of the RNAi machinery (Kakumani et al., 2013). As a result, GRP75 was isolated and shown to interact with an auxiliary component of the Dicer complex. GRP75, also known as HSPA9 or mortalin, belongs to the highly conserved 70 $\mathrm{kDa}$ heat shock protein (HSP70) family (Daugaard et al., 2007). In ectopic GRP75-expressing cells, the processing of miRNA precursors into mature miRNAs was enhanced. In addition, knockdown of GRP75 in Huh7 cells led to an increase of viral RNA level, but the effect of GRP75 on extracellular viral yield was not evaluated. Based on the role of GRP75 in miRNA processing and the modulation of DENV RNA levels, the authors proposed that GRP75 might down-regulate DENV replication by promoting the mature processing of the antiviral miRNA hsa-mir-126 (Kakumani et al., 2016). This hypothesis needs to be verified, as GRP75 has a general role in the processing of miRNAs (including both antiviral and proviral miRNAs), while the study did not demonstrate that the role of GRP75 was specifically mediated by its effect on has-mir-126.

Eukaryotic initiation factor $4 A I$ (eIF4AI). The eIF4AI protein belongs to the DEAD(Asp-Glu-Ala-Asp)-box RNA helicase family. Structurally, eIF4AI is composed of an N-terminal ATP-binding domain and a C-terminal helicase domain (Wei et al., 2001). Using a tandem affinity purification (TAP) assay, Chen et al. identified eIF4AI as a cellular protein that interacts with the DENV NS4A protein (Chen et al., 2015). Knockdown of eIF4AI led to an increased accumulation of viral proteins, but it barely affected viral RNA levels, suggesting that the antiviral effect of eIF4AI might be the result of the inhibition of viral protein accumulation. Further research showed that the knockdown of eIF4AI led to lower phosphorylation levels of the protein kinase regulated by double-stranded RNA (protein kinase R) and its substrate eIF $2 \alpha$, which can inhibit protein synthesis. Therefore, the antiviral effect of eIF4AI might be dependent on its role in promoting PKR activation (Chen et al., 2015).

$D E A D$-box helicase 3 (DDX3). DDX3, which is also known as DDX3X, is a member of a family of putative RNA helicases. DDX3 is involved in various aspects of RNA metabolism, including transcriptional regulation, splicing, mRNA export, ribosome biogenesis, and translational regulation (Rocak and Linder, 2004). DDX3 has been implicated in the inhibition of hepatitis B virus and vesicular stomatitis virus infections (Wang et al., 2009; Oshiumi et al., 2010). Li's group screened for the host factors involved in DENV replication by using RNAi to knockdown 40 members of the DEAD-box family. DDX3 was found to be an antiviral factor. In HEK293T and A549 cells infected with DENV, transcription level of DDX3 was significantly upregulated. Moreover, overexpression of DDX3 led to an increase in IFN- $\beta$ tran- 
scription and activation of NF- $\kappa \mathrm{B}$ and interferon regulatory transcription factor 3 (IRF3) (Li et al., 2015). Combined with the previous observation that DDX3 binds to the IFN adaptor IFN- $\beta$ promoter stimulator 1 (IPS- 1 ) to regulate downstream signaling, it appears that DDX3 is involved in the recognition of viral RNA or the regulation of IPS-1 functions, and the promotion of the IFN response to combat DENV infection (Oshiumi et al., 2010).

\section{HOST miRNAs AND DENV}

Research has shown that miRNAs play many roles in diverse biological processes, including development, apoptosis, and tumorigenesis, by binding to the 3'-UTR of target mRNAs to induce their degradation or suppress their translation (Miesen et al., 2016). In recent years, studies have gradually identified more miRNAs that are involved in viral infection and replication by targeting host or viral proteins. A microarray profiling study using the blood of patients with acute DENV infections showed that the expression levels of 348 miRNAs were altered in response to DENV infection. Moreover, the levels of some miRNAs were different between mild DENV infections and severe diseases, so these miRNAs are potential biomarkers for the clinic diag-nosis of DENV diseases (Tambyah et al., 2016).

\section{Proviral miRNAs}

$m i R-146 a$. The proviral miR-146a is induced by multiple viruses and viral proteins, including human $\mathrm{T}$ cell leukemia virus type I, human immunodeficiency virus, vesicular stomatitis virus, Epstein-Barr virus latent membrane protein 1, and the Kaposi's sarcoma-associated herpesvirus K13 protein (Rusca and Monticelli, 2011). In DENV2-infected monocytes, the expression of miR-146a was significantly increased (Wu et al., 2013). Overexpression of miR-146 led to an increase in viral replication and a reduction in IFN- $\beta$ production. In contrast, inhibition of miR-146 reduced viral replication and enhanced the production of IFN- $\beta$. This suggests that the antiviral activity of miR-146a might be correlated with IFN induction. Further research showed that TRAF6, an important adaptor in the innate immune signaling pathway, is one of miR-146a targets. Complementation with TRAF6 reversed the effects of miR-146a on viral replication and IFN- $\beta$ production. Thus, it appears that miR146a functions as a proviral factor by targeting the host IFN defense system (Wu et al., 2013).

$m i R-281$. Proviral miR-281 was found to be highly expressed in the midgut of both sugar- and blood-fed female Aedes albopictus mosquitoes, which are vectors of DENV (Gu et al., 2013). The expression of miR-281 was induced in mosquito cells or midguts at 4 or 7 days after infection. Overexpression of miR-281 promoted viral replication in C6/36 insect cells, and miR-281 knockdown reduced the viral RNA levels in vivo in Ae. albopictus mosquitoes. In addition, miR-281 was shown to potentially target the 5'-UTR of the DENV-2 genome RNA, which requires further investigation (Zhou et al., 2014).

\section{Antiviral miRNAs}

Receptor-activated miR-30e (miR-30e*). Expression of members of the miR-30 family has been associated with type I IFN and hepatitis $\mathrm{C}$ virus infections (Pedersen et al., 2007; Carolin et al., 2010). Zhu et al. found that the expression of miR-30e* was upregulated in DENV-infected HeLa cells. Overexpression of miR-30e* suppressed the viral replication, and inhibition of miR-30* resulted in enhanced viral replication. The authors demonstrated that miR-30* targets the $3^{\prime}$-UTR of I $\kappa-B \alpha$, an inhibitory molecule in the innate immune signaling pathway, leading to the activation of NF- $\mathrm{KB}$. The antiviral effect of miR-30* is exerted via upregulation of the transcription of antiviral IFN- $\beta$ and downstream IFN-stimulated genes, such as 2'-5'-oligoadenylate synthetase 1 (OAS1), myxovirus resistance A (MxA), and interferon induced transmembrane protein 1 (IFITM1) (Zhu et al., 2014).

Lethal-7c (let-7c). The let-7 family is highly conserved in multiple organisms and it belongs to a group of 13 miRNAs that have been shown to be inhibitors of processes involved in cell proliferation (Boyerinas et al., 2010). In the miRNA profiling assay conducted by Yocupicio-Monroy's group, several miRNAs including let-7c, miRNA-92*, miRNA-149*, and miR-181c* were increased in response to DENV infection (EscaleraCueto et al., 2015). Among these miRNAs, let-7c overexpression suppressed the replication of DENV, which was restored by the expression of BTB and CNC homology 1 (BACH1), one of let-7c targets. The study revealed that $\mathrm{BACH} 1$ expression was downregulated in DENV infections, which correlated with the upregulation of let-7c. As HO-1 might be regulated by BACH1, and its expression was upregulated in DENV-infected cells, the authors proposed that the antiviral role of let- $7 \mathrm{c}$ may be exerted by targeting BACH1, leading to the induction of the anti-inflammatory and anti-oxidative HO-1 (Escalera-Cueto et al., 2015).

miR-223. In an miRNA microarray screen, An's group found that the expression of miR-223 was downregulated by DENV2 infection (Wu et al., 2014). In cells expressing less miR-223, the viral yield was increased. Using a complementation assay, the microtubule-destabilizing protein stathmin 1 (STMN1) was demonstrated to be 
one of miR-223 targets, and the antiviral effect of miR223 was shown to be mediated by targeting STMN1. Although STMN1 expression was found to be upregulated in DENV-infected cells, the detailed mechanism behind STMN1-induced promotion of DENV2 infection needs to be further investigated (Wu et al., 2014).

$m i R-133 a$. Using a bioinformatic analysis, the host miR133 a was found to potentially target the 3 '-UTR of all four DENV serotypes (Castillo et al., 2016). Castillo et al . revealed that miR-133a overexpression suppressed DENV replication. PTB, the proviral protein described earlier, has been predicted to be a potential target of miR-133a (Fred et al., 2010). The authors showed that the overexpression of miR-133a in Vero cells led to a significantly lower level of PTB protein. Expression of miR-133a was downregulated by the 3'-UTR of DENV, while expression of PTB was increased in the early stage of DENV infections (12-18 hours after infection). However, a complementation assay was not carried out to confirm that the antiviral effect of miR-133a is mediated by targeting PTB (Castillo et al., 2016).

\section{CONCLUDING REMARKS}

As the number of people at risk of DENV infection is increasing due to global warming, expansion of urban populations and global travel(Diamond and Pierson, 2015), much effort has been put into elucidating the mechanisms behind DENV replication, dissemination, and pathogenesis. Although significant progress has been made, there are still many issues that require further investigation: more of the host factors involved in the viral life cycle need to be identified; the precise mechanisms of many known host factors (especially miRNAs) need to be confirmed; the correlation between miRNAs and immunopathogensis (e.g., virus-induced inflammation of the endothelium) needs to be investigated (Aloia et al., 2015); and, importantly, the functions of many host factor's remain to be assessed in vivo due to the current lack of an appropriate animal model for the study of DENV infection. Resolving these issues will not only help us to better understand the basic biology of DENV, but it will also provide novel therapeutic targets for antiDENV drugs.

\section{ACKNOWLEDGMENTS}

This work was supported by the National Natural Science Foundation of China (81371794) and Guangdong Natural Science Foundation (2014A030311007).

\section{COMPLIANCE WITH ETHICS GUIDELINES}

The authors declare that they have no conflict of interest.
This article does not contain any studies with human or animal subjects performed by any of the authors.

\section{REFERENCES}

Acosta EG, Castilla V, Damonte EB. 2008. Functional entry of dengue virus into Aedes albopictus mosquito cells is dependent on clathrin-mediated endocytosis. J Gen Virol, 89: 474-484.

Acosta EG, Kumar A, Bartenschlager R. 2014. Revisiting dengue virus-host cell interaction: new insights into molecular and cellular virology. Adv Virus Res, 88: 1-109.

Agis-Juarez RA, Galvan I, Medina F, Daikoku T, Padmanabhan R, Ludert JE, del Angel RM. 2009. Polypyrimidine tract-binding protein is relocated to the cytoplasm and is required during dengue virus infection in Vero cells. J Gen Virol, 90: 28932901.

Aloia AL, Abraham AM, Bonder CS, Pitson SM, Carr JM. 2015. Dengue Virus-Induced Inflammation of the Endothelium and the Potential Roles of Sphingosine Kinase-1 and MicroRNAs. Mediators Inflamm, 2015: 509306.

Borrego F. 2013. The CD300 molecules: an emerging family of regulators of the immune system. Blood, 121: 1951-1960.

Boyerinas B, Park SM, Hau A, Murmann AE, Peter ME. 2010. The role of let-7 in cell differentiation and cancer. Endocr Relat Cancer, 17: F19-36.

Brasier AR, Zhao Y, Wiktorowicz JE, Spratt HM, Nascimento EJM, Cordeiro MT, Soman KV, Ju H, Recinos A, Stafford S, Wu Z, Marques ETA, Vasilakis N. 2015. Molecular classification of outcomes from dengue virus -3 infections. J Clin Virol, 64: 97-106.

Brunetti JE, Scolaro LA, Castilla V. 2015. The heterogeneous nuclear ribonucleoprotein $\mathrm{K}(\mathrm{hnRNP} \mathrm{K})$ is a host factor required for dengue virus and Junín virus multiplication. Virus Res, 203: 84-91.

Carnec X, Meertens L, Dejarnac O, Perera-Lecoin M, Hafirassou ML, Kitaura J, Ramdasi R, Schwartz O, Amara A. 2015. The Phosphatidylserine and Phosphatidylethanolamine Receptor CD300a Binds Dengue Virus and Enhances Infection. J Virol, 90: 92-102.

Carolin S, Pompea Z, Jacopo V, Carla S, Delia R, Gloria T, Elisabetta R, Anthony AJ, Eligio P, Guido A. 2010. Differential expression of interferon-induced microRNAs in patients with chronic hepatitis $\mathrm{C}$ virus infection treated with pegylated interferon alpha. Virol J, 7: 311.

Casseb SM, Simith DB, Melo KF, Mendonca MH, Santos AC, Carvalho VL, Cruz AC, Vasconcelos PF. 2016. Drosha, DGCR8, and Dicer mRNAs are down-regulated in human cells infected with dengue virus 4, and play a role in viral pathogenesis. Genet Mol Res, 15: gmr.15027891.

Castillo JA, Castrillon JC, Diosa-Toro M, Betancur JG, St Laurent G, 3rd, Smit JM, Urcuqui-Inchima S. 2016. Complex interaction between dengue virus replication and expression of miRNA133a. BMC Infect Dis, 16: 29.

Chen X, Xia J, Zhao Q, Wang Y, Liu J, Feng L, He J, Zhang P. 2015. Eukaryotic initiation factor 4AI interacts with NS4A of Dengue virus and plays an antiviral role. Biochem Biophys Res Commun, 461: 148-153.

Daugaard M, Rohde M, Jaattela M. 2007. The heat shock protein 70 family: Highly homologous proteins with overlapping and distinct functions. FEBS Lett, 581: 3702-3710.

Diamond MS, Pierson TC. 2015. Molecular Insight into Dengue Virus Pathogenesis and Its Implications for Disease Control. Cell, 162: 488-492.

Dixit E, Boulant S, Zhang Y, Lee AS, Odendall C, Shum B, Haco- 
hen N, Chen ZJ, Whelan SP, Fransen M, Nibert ML, SupertiFurga G, Kagan JC. 2010. Peroxisomes are signaling platforms for antiviral innate immunity. Cell, 141: 668-681.

Escalera-Cueto M, Medina-Martinez I, del Angel RM, BerumenCampos J, Gutierrez-Escolano AL, Yocupicio-Monroy M. 2015. Let-7c overexpression inhibits dengue virus replication in human hepatoma Huh-7 cells. Virus Res, 196: 105-112.

Fred RG, Bang-Berthelsen CH, Mandrup-Poulsen T, Grunnet LG, Welsh N. 2010. High glucose suppresses human islet insulin biosynthesis by inducing miR-133a leading to decreased polypyrimidine tract binding protein-expression. PLoS One, 5: e10843.

Green AM, Beatty PR, Hadjilaou A, Harris E. 2014. Innate immunity to dengue virus infection and subversion of antiviral responses. J Mol Biol, 426: 1148-1160.

Gu J, Hu W, Wu J, Zheng P, Chen M, James AA, Chen X, Tu Z. 2013. miRNA Genes of an Invasive Vector Mosquito, Aedes albopictus. PLoS One, 8: e67638.

Gubler DJ. 1998. Dengue and Dengue Hemorrhagic Fever. Clin Microbiol Rev, 11: 480-496.

Han SP, Tang YH, Smith R. 2010. Functional diversity of the hnRNPs: past, present and perspectives. Biochem J, 430: 379-392.

Hidari KI, Suzuki T. 2011. Dengue virus receptor. Trop Med Health, 39: 37-43.

Huerta V, Toledo P, Fleitas N, Martin A, Pupo D, Yero A, Sarria M, Sanchez A, Besada V, Ramos Y, Marquez G, Guirola O, Chinea G. 2014. Receptor-activated human alpha2-macroglobulin interacts with the envelope protein of dengue virus and protects virions from temperature-induced inactivation through multivalent binding. J Gen Virol, 95: 2668-2676.

Iglesias NG, Mondotte JA, Byk LA, De Maio FA, Samsa MM, Alvarez C, Gamarnik AV. 2015. Dengue Virus Uses a Non-Canonical Function of the Host GBF1-Arf-COPI System for Capsid Protein Accumulation on Lipid Droplets. Traffic, 16: 962-977.

Kakumani PK, Medigeshi GR, Kaur I, Malhotra P, Mukherjee SK, Bhatnagar RK. 2016. Role of human GRP75 in miRNA mediated regulation of dengue virus replication. Gene, 586: 7-11.

Kakumani PK, Ponia SS, S RK, Sood V, Chinnappan M, Banerjea AC, Medigeshi GR, Malhotra P, Mukherjee SK, Bhatnagar RK. 2013. Role of RNA interference (RNAi) in dengue virus replication and identification of NS4B as an RNAi suppressor. J Virol, 87: 8870-8883.

Krishnan MN, Garcia-Blanco MA. 2014. Targeting host factors to treat West Nile and dengue viral infections. Viruses, 6: 683-708.

Li G, Feng T, Pan W, Shi X, Dai J. 2015. DEAD-box RNA helicase DDX3X inhibits DENV replication via regulating type one interferon pathway. Biochem Biophys Res Commun, 456: 327-332.

Liu B, Li NL, Wang J, Shi PY, Wang T, Miller MA, Li K. 2014. Overlapping and distinct molecular determinants dictating the antiviral activities of TRIM56 against flaviviruses and coronavirus. J Virol, 88: 13821-13835.

Ma H, Dang Y, Wu Y, Jia G, Anaya E, Zhang J, Abraham S, Choi JG, Shi G, Qi L, Manjunath N, Wu H. 2015. A CRISPR-Based Screen Identifies Genes Essential for West-Nile-Virus-Induced Cell Death. Cell Rep, 12: 673-683.

Marceau CD, Puschnik AS, Majzoub K, Ooi YS, Brewer SM, Fuchs G, Swaminathan K, Mata MA, Elias JE, Sarnow P, Carette JE. 2016. Genetic dissection of Flaviviridae host factors through genome-scale CRISPR screens. Nature, 535: 159-163.

Marques RE, Guabiraba R, Del Sarto JL, Rocha RF, Queiroz AL,
Cisalpino D, Marques PE, Pacca CC, Fagundes CT, Menezes GB, Nogueira ML, Souza DG, Teixeira MM. 2015. Dengue virus requires the $\mathrm{CC}$-chemokine receptor CCR5 for replication and infection development. Immunology, 145: 583-596.

Meertens L, Carnec X, Lecoin MP, Ramdasi R, Guivel-Benhassine F, Lew E, Lemke G, Schwartz O, Amara A. 2012. The TIM and TAM families of phosphatidylserine receptors mediate dengue virus entry. Cell Host Microbe, 12: 544-557.

Michael JI, Salvatore Vp. 1981. Clearance and Binding of Two Electrophoretic "Fast" Forms of Human a2-Macroglobulin. J Biol Chem, 256: 8134-8139.

Miesen P, Ivens A, Buck AH, van Rij RP. 2016. Small RNA Profiling in Dengue Virus 2-Infected Aedes Mosquito Cells Reveals Viral piRNAs and Novel Host miRNAs. PLoS Negl Trop Dis, 10: e0004452.

Oppermann M. 2004. Chemokine receptor CCR5: insights into structure, function, and regulation. Cell Signal, 16: 1201-1210.

Oshiumi H, Sakai K, Matsumoto M, Seya T. 2010. DEAD/H BOX 3 (DDX3) helicase binds the RIG-I adaptor IPS-1 to up-regulate IFN-beta-inducing potential. Eur J Immunol, 40: 940-948.

Paine A, Eiz-Vesper B, Blasczyk R, Immenschuh S. 2010. Signaling to heme oxygenase-1 and its anti-inflammatory therapeutic potential. Biochem Pharmacol, 80: 1895-1903.

Pedersen IM, Cheng G, Wieland S, Volinia S, Croce CM, Chisari FV, David M. 2007. Interferon modulation of cellular micro RNAs as an antiviral mechanism. Nature, 449: 919-922.

Perreira JM, Aker AM, Savidis G, Chin CR, McDougall WM, Portmann JM, Meraner P, Smith MC, Rahman M, Baker RE, Gauthier A, Franti M, Brass AL. 2015. RNASEK Is a V-ATP ase-Associated Factor Required for Endocytosis and the Replication of Rhinovirus, Influenza A Virus, and Dengue Virus. Cell Rep, 12: 850-863.

Rajsbaum R, Garcia-Sastre A, Versteeg GA. 2014. TRIMmunity: the roles of the TRIM E3-ubiquitin ligase family in innate antiviral immunity. J Mol Biol, 426: 1265-1284.

Ramaen O, Joubert A, Simister P, Belgareh-Touze N, OlivaresSanchez MC, Zeeh JC, Chantalat S, Golinelli-Cohen MP, Jackson CL, Biou V, Cherfils J. 2007. Interactions between conserved domains within homodimers in the BIG1, BIG2, and GBF1 Arf guanine nucleotide exchange factors. J Biol Chem, 282: 28834-28842.

Repeke CE, Ferreira SB, Jr., Claudino M, Silveira EM, de Assis GF, Avila-Campos MJ, Silva JS, Garlet GP. 2010. Evidences of the cooperative role of the chemokines CCL3, CCL4 and CCL5 and its receptors CCR $1+$ and CCR5+ in RANKL+ cell migration throughout experimental periodontitis in mice. Bone, 46: 1122-1130.

Rocak S, Linder P. 2004. DEAD-box proteins: the driving forces behind RNA metabolism. Nat Rev Mol Cell Biol, 5: 232-241.

Rusca N, Monticelli S. 2011. MiR-146a in Immunity and Disease. Mol Biol Int, 2011: 437301.

Salazar MI, del Angel RM, Lanz-Mendoza H, Ludert JE, PandoRobles V. 2014. The role of cell proteins in dengue virus infection. J Proteomics, 111: 6-15.

Samsa MM, Mondotte JA, Caramelo JJ, Gamarnik AV. 2012. Uncoupling cis-Acting RNA elements from coding sequences revealed a requirement of the $\mathrm{N}$-terminal region of dengue virus capsid protein in virus particle formation. J Virol, 86: 10461058.

Savidis G, McDougall WM, Meraner P, Perreira JM, Portmann JM, Trincucci G, John SP, Aker AM, Renzette N, Robbins DR, Guo Z, Green S, Kowalik TF, Brass AL. 2016. Identification of Zika Virus and Dengue Virus Dependency Factors using Functional Genomics. Cell Rep, 16: 232-246. 
Tambyah PA, Ching CS, Sepramaniam S, Ali JM, Armugam A, Jeyaseelan K. 2016. MicroRNA expression in blood of dengue patients. Ann Clin Biochem, 53: 466-476.

Tseng CK, Lin CK, Wu YH, Chen YH, Chen WC, Young KC, Lee JC. 2016. Human heme oxygenase 1 is a potential host cell factor against dengue virus replication. Sci Rep, 6: 32176.

Wang H, Kim S, Ryu WS. 2009. DDX3 DEAD-Box RNA helicase inhibits hepatitis $\mathrm{B}$ virus reverse transcription by incorporation into nucleocapsids. J Virol, 83: 5815-5824.

Wei L, Natalie R-S, Christopher GP, Graham JB. 2001. Cleavage of translation initiation factor 4AI (eIF4AI) but not eIF4AII by foot-and-mouth disease virus $3 \mathrm{C}$ protease: identiccation of the eIF4AI cleavage site. FEBS Lett, 507: 1-5.

Welsch S, Miller S, Romero-Brey I, Merz A, Bleck CK, Walther P, Fuller SD, Antony C, Krijnse-Locker J, Bartenschlager R. 2009. Composition and three-dimensional architecture of the dengue virus replication and assembly sites. Cell Host Microbe, 5: 365-375.

Wu N, Gao N, Fan D, Wei J, Zhang J, An J. 2014. miR-223 inhibits dengue virus replication by negatively regulating the microtubule-destabilizing protein STMN1 in EAhy926 cells. Microbes Infect, 16: 911-922.

Wu S, He L, Li Y, Wang T, Feng L, Jiang L, Zhang P, Huang X. 2013. miR-146a facilitates replication of dengue virus by dampening interferon induction by targeting TRAF6. J Infect, 67: $329-341$.
You J, Hou S, Malik-Soni N, Xu Z, Kumar A, Rachubinski RA, Frappier L, Hobman TC. 2015. Flavivirus Infection Impairs Peroxisome Biogenesis and Early Antiviral Signaling. J Virol, 89: 12349-12361.

Yu IM, Zhang W, Holdaway HA, Li L, Kostyuchenko VA, Chipman PR, Kuhn RJ, Rossmann MG, Chen J. 2008. Structure of the immature dengue virus at low $\mathrm{pH}$ primes proteolytic maturation. Science, 319: 1834-1837.

Zaitseva E, Yang ST, Melikov K, Pourmal S, Chernomordik LV. 2010. Dengue virus ensures its fusion in late endosomes using compartment-specific lipids. PLoS Pathog, 6: e1001131.

Zhang R, Miner JJ, Gorman MJ, Rausch K, Ramage H, White JP, Zuiani A, Zhang P, Fernandez E, Zhang Q, Dowd KA, Pierson TC, Cherry S, Diamond MS. 2016. A CRISPR screen defines a signal peptide processing pathway required by flaviviruses. Nature, 535: 164-168.

Zhou Y, Liu Y, Yan H, Li Y, Zhang H, Xu J, Puthiyakunnon S, and Chen X. 2014. miR-281, an abundant midgut-specific miRNA of the vector mosquitoAedes albopictusenhances dengue virus replication. Parasites Vectors, 7: 488.

Zhu X, He Z, Hu Y, Wen W, Lin C, Yu J, Pan J, Li R, Deng H, Liao S, Yuan J, Wu J, Li J, Li M. 2014. MicroRNA-30e* Suppresses Dengue Virus Replication by Promoting NF-kB-Dependent IFN Production. PLOS Neglected Tropical Diseases, 8: e3088. 